\title{
SSH1 expression is associated with gastric cancer progression and predicts a poor prognosis
}

\author{
Yusufu Maimaiti ${ }^{1 \dagger}$, Maimaitiaili Maimaitiming ${ }^{1 \dagger}$, Yiliang Li ${ }^{1}$, Saifuding Aibibula', Azatijiang Ainiwaer ${ }^{1}$, Aikebaier Aili ${ }^{1}$, \\ Zhenzhu Sun ${ }^{2}$ and Kelimu Abudureyimu ${ }^{1 *}$ (I)
}

\begin{abstract}
Background: Slingshot homolog-1 (SSH1) plays an important role in pathological processes, including in the occurrence and development of tumours. The purpose of this study was to determine whether SSH1 is a key biomarker with prognostic value for survival in patients with gastric cancer.

Methods: We performed immunohistochemistry $(\mathrm{IHC})$ on tissue microarrays containing 100 gastric cancer specimens to evaluate SSH1 protein expression. The association of pathological characteristics with cumulative survival was determined by Kaplan-Meier analysis. A Cox proportional hazards model was generated in the multi-factorial survival analysis to identify univariate prognostic factors of GC.

Results: $\mathrm{SSH} 1$ expression level in gastric cancer tissues was significantly associated with lymph node metastasis $(P=0.032)$. Additionally, multivariate regression analysis clearly indicated that $\mathrm{SSH} 1$ expression was significantly correlated with poor clinical outcomes of patients with gastric cancer $(P=0.016)$. Multivariate analyses showed that SSH1 was the best predictor of poor prognosis in patients with gastric cancer $(P=0.030)$.

Conclusions: $\mathrm{SSH} 1$ expression is associated with gastric cancer progression and predicts a poor prognosis. SSH1 may play an important role in the development of gastric cancer, and it is a promising target for prevention and/ or treatment of gastric cancer.
\end{abstract}

Keywords: SSH1, Gastric cancer, Progression, Survival analysis

\section{Background}

Gastric cancer (GC) is a common type of malignancy and the second leading cause of cancer-related death in China [1]. Unfortunately, the incidence of GC has been increasing in recent years; there were 1313 thousand incident cases and 819 thousand deaths in 2015 [2]. Therapeutic approaches to GC include surgical treatment, chemotherapy, radiotherapy, and gene therapy. However, the prognosis for GC patients remains poor [3],patients with early-stage GC have nonspecific symptoms, and lymph node invasion or distant metastasis has already occurred by the time they are diagnosed [4].

\footnotetext{
*Correspondence: klm6075@163.com

${ }^{\dagger}$ Equal contributors

'Department of General Surgery (Research Institute of Minimally Invasive), People's Hospital of Xinjiang Uygur Autonomous Region, Urumqi 830000, China

Full list of author information is available at the end of the article
}

Therefore, it is important to identify biomarkers that are related to early diagnosis and prognosis.

Cancer cell migration and invasion lead to GC metastasis, which accounts for most cancer-related deaths. The metastasis of GC is a complex, multistep pathological process that involves several signalling pathways. Emerging evidence has shown that the balance between protein phosphorylation and dephosphorylation is crucial in the regulation of cell signalling, and failure to maintain an appropriate balance is known to play a critical role in cancer development [4].

In mammalian cells, SSH phosphatases are encoded by three genes (SSH1, SSH2, and SSH3) that encode members of a family of serine/threonine protein kinases that dephosphorylate the phospho-serine residue of cofilin $[5,6]$ and reactivate cofilin through phosphorylation at serine-3 [7]. SSH1 was found to be overexpressed in 
pancreatic cancer (PC) and to contribute to tumour cell migration [8]. Recent studies have demonstrated that SSH1 is a key regulator of vascular smooth muscle cell migration [9]. When cells are stimulated by growth factors or chemokines, SSH1 accumulates in the F-actinrich lamellipodium and likely plays a critical role in polarized cell migration by maintaining the local activation of cofilin and the rapid turnover of actin filaments at the leading edge of the migrating cell [10]. In addition, there is evidence suggesting that SSH1 plays an important role in cancer development. However, there is currently no evidence suggesting that SSH1 expression correlates with GC prognosis. Thus, studies are needed to determine whether SSH1 is a prognostic biomarker for GC. We designed a tissue microarray (TMA) covering 100 patients with 8 years of follow up. We performed IHC analysis to investigate the correlation between SSH1 expression and clinico-pathological characteristics and to determine whether SSH1 can serve as a prognostic marker.

\section{Methods}

This study was approved by the hospital ethics committee, and written informed consent was received from the patients. We used a retrospective design to analyse GC tissue samples and all patients with GC had pathologically proven disease and underwent surgical operation between July 2006 and April 2007. All patients were followed up for 19-105 months (average, 49.74 months). The GC tissue and adjacent-to-carcinoma TMAs with a total of 100 cases of GC and 80 cases of adjacent-tocarcinoma paraffin-embedded tissue specimens were purchased from the National Engineering Center for Bio Chips, Shanghai, People's Republic of China. Complete histological data for the patients with GC were available.

The patients' clinical characteristics, such as gender, age, tumour location, tumour size, pathological type, pathological grading, tumour infiltration, lymph node metastasis, total lymph nodes dissected, total number of positive lymph nodes (pathology), and distant metastasis, were obtained from their medical records (Table 1). Metastasis status was expressed using the pathologic stage of the disease determined according to the seventh edition of the American Joint Committee on Cancer (AJCC)/International Union Against Cancer TNM classification system. TMA and IHC staining of SSH1 in GC tissue (100 cases) and adjacent-to-carcinoma tissue (80 cases) was performed in our study (Fig. 1a).

\section{IHC and scoring}

Immunohistochemistry (IHC) staining was used to detect SSH1 expression in the GC and adjacent-tocarcinoma TMA (Fig. 1b \& c). To evaluate SSH1 expression, we used a SSH1-specific antibody from Abcam
Table 1 Clinico-pathological features of the 100 patients with GC

\begin{tabular}{|c|c|c|}
\hline Clinico-pathologic features & Cancer & Adjacent to carcinoma \\
\hline $\mathrm{SSH1}(+)$ & 66 & 66 \\
\hline SSH1 (-) & 34 & 14 \\
\hline \multicolumn{3}{|l|}{ Age (years) } \\
\hline$\leq 55$ & 21 & 17 \\
\hline$>55$ & 79 & 63 \\
\hline \multicolumn{3}{|l|}{ Gender } \\
\hline Male & 64 & 47 \\
\hline Female & 36 & 33 \\
\hline \multicolumn{3}{|l|}{ Location } \\
\hline Cardiac & 11 & 10 \\
\hline Fundus & 8 & 8 \\
\hline Corpora & 18 & 13 \\
\hline Sinuses & 57 & 46 \\
\hline Total & 6 & 3 \\
\hline \multicolumn{3}{|l|}{ Pathological type } \\
\hline Glandular & 64 & 50 \\
\hline Signet ring cell & 12 & 9 \\
\hline Mucinous cell & 11 & 9 \\
\hline Undifferentiation & 12 & 11 \\
\hline Missing & 1 & 1 \\
\hline \multicolumn{3}{|l|}{ Lymph node metastasis } \\
\hline Positive & 79 & 63 \\
\hline Negative & 21 & 17 \\
\hline \multicolumn{3}{|l|}{ Tumour infiltration } \\
\hline $\mathrm{T} 1$ & 6 & 5 \\
\hline $\mathrm{T} 2$ & 7 & 4 \\
\hline T3 & 66 & 55 \\
\hline T4 & 20 & 15 \\
\hline Missing & 1 & 1 \\
\hline \multicolumn{3}{|l|}{ Size } \\
\hline$\geq 5 \mathrm{~cm}$ & 59 & 45 \\
\hline$<5 \mathrm{~cm}$ & 39 & 35 \\
\hline Missing & 2 & 0 \\
\hline \multicolumn{3}{|l|}{ AJCC staging } \\
\hline Stage I & 10 & 7 \\
\hline Stage II & 33 & 29 \\
\hline Stage III & 49 & 36 \\
\hline Stage IV & 7 & 7 \\
\hline Missing & 1 & 1 \\
\hline
\end{tabular}

(ab76943; Cambridge, UK) at a dilution of 1:1000, based on the manufacturer's protocol. Two experienced pathologists who were blinded to the patients' clinical information independently evaluated and recorded the IHC results. Brown-yellow staining indicated SSH1- 

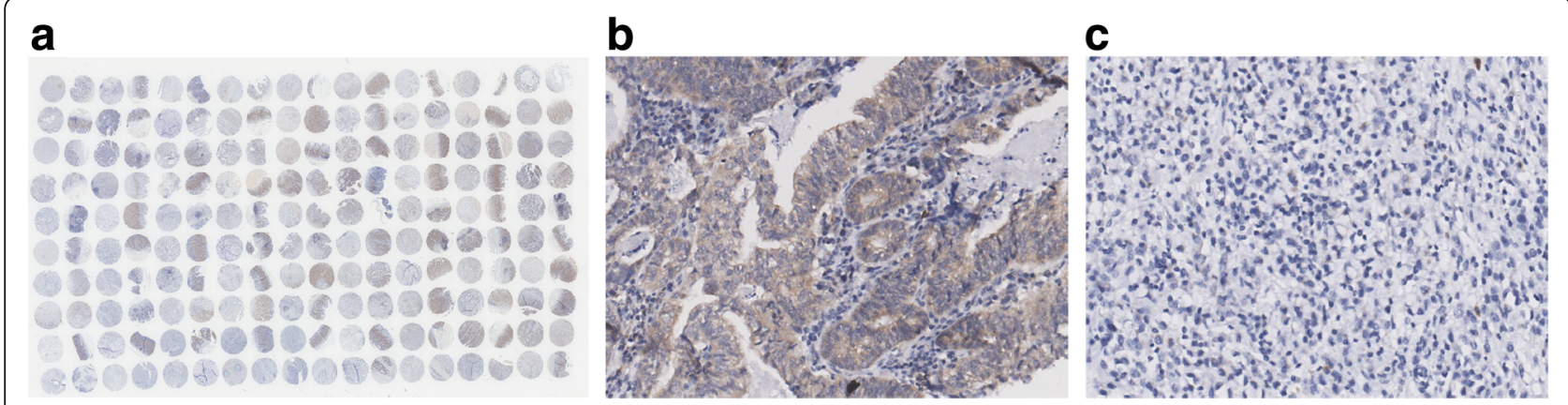

Fig. 1 Tissue microarray and IHC staining of SSH1 in GC tissues. a Tissue microarray (100 cases of GC tissue and 80 cases of adjacent-tocarcinoma tissue), 1× magnification. b Positive expression of SSH1 in cancer tissues; 200× magnification. c Negative expression of SSH1 in cancer tissues; 200× magnification

positive expression. According to the staining intensity and percentage of positive cells, the tissue samples were comprehensively scored. The SSH1 staining intensity in the tumour cells was graded as $0-3$, and the percentage of SSH1-positive cells was graded according to the proportion of positive cells using a $0-4$ grading scale $(0$ : 0\%-5\%; 1: 6\%-25\%; 2: 26\%-50\%; 3: 51\%-75\%; 4: 76\%$100 \%$ ). The final SSH1 expression score (positive or negative) was calculated as the sum of both grades (negative: total grade $=0-3$; positive: total grade $=4-7$ ) .

\section{Survival and statistical analysis Methods}

All slides were scanned using an Aperio Scan Scope slide scanner, and images of representative areas were obtained using Image Scope software (Aperio) and Adobe Illustrator. The primary clinical and histopathological data were compiled using EpiData software (version 3.1; EpiData Association, Odense, Denmark).

\section{Statistical analysis}

The Chi squared test and Fisher's exact probability method were adopted to compare SSH1 expression levels and clinico-pathological parameters in the 100 patients with GC. The Kaplan-Meier survival curve method and log-rank test were applied to compare the survival rate between groups. A Cox proportional hazards model was generated in the multi-factorial survival analysis to identify univariate prognostic factors. SPSS software (version 22.0; SPSS Inc., Chicago, IL, USA) was used to analyse the data. All tests were two-sided, and $P$ values $<0.05$ indicated statistical significance.

\section{Results}

The detailed clinico-pathological characteristics of these 100 patients are provided in Table 1 . According to the total lymph nodes dissected and positive lymph nodes results, which were confirmed by pathology, we calculated the positive lymph node ratio, which defined as ratio of positive lymph nodes to all lymph nodes removed, is a powerful prognostic factor in cancer [11], and identified 43 cases with a positive lymph node ratio $\geq 0.5$ and 57 cases with a positive lymph node ratio < 0.5. Tumour infiltration was at T1 in 8 cases, T2 in 7 cases, and T3 in 64 cases and T4 in 20 cases; 1 case was unknown. Lymph node metastasis (LNM) was positive in 79 cases, and 21 cases had no LNM. Regarding pathological grade (I-IV), there were 15 cases of grade II, 74 cases of grade III (II-III, III), and 11 cases of grade IV (III-IV). According to the seventh edition of the AJCC staging system, 10 cases were phase I, 33 were phase II, 49 were phase III, 7 were phase IV, and 1 was unknown.

\section{Association between SSH1 expression in GC and adjacent-} to-carcinoma tissues and clinico-pathological features of GC In our study, 66 cases of GC tumour tissue (66\%) and 66 cases of adjacent-to-carcinoma tissue (82.5\%) showed positive SSH1 expression, indicating that SSH1 is expressed at a high frequency. The difference in SSH1 expression between GC and adjacent-tocarcinoma tissues was not statistically significant $(P=$ 0.769; Table 2). We investigated the relationship between SSH1 expression levels in GC and adjacent-tocarcinoma tissues and clinico-pathological features of GC (Table 3), and no significant correlation was found between SSH1 expression in GC tissue and sex, age, tumour size, tumour location, pathological type, pathological grade, positive lymph node ratio or AJCC stage (all $P>0.05$, Table 3 ); there was a correlation with only lymphatic metastasis $(P=0.032$; Table 3$)$.

Table 2 Relationship between cancer tissues and adjacent-tocarcinoma tissues

\begin{tabular}{|c|c|c|c|c|c|}
\hline & & \multicolumn{2}{|l|}{ Cancer } & \multirow[t]{2}{*}{ Correlation } & \multirow[t]{2}{*}{$P$-value } \\
\hline & & $\overline{\mathrm{SSH} 1(+)}$ & $\overline{\mathrm{SSH} 1(-)}$ & & \\
\hline \multirow{2}{*}{$\begin{array}{l}\text { Adjacent to } \\
\text { Carcinoma }\end{array}$} & $\mathrm{SSH1}(+)$ & 25 & 41 & \multirow[t]{2}{*}{-0.039} & \multirow[t]{2}{*}{0.769} \\
\hline & SSH1(-) & 6 & 8 & & \\
\hline
\end{tabular}


Table 3 Association between SSH1 expression in GC and adjacent-to-carcinoma tissues and clinico-pathological features of GC

\begin{tabular}{|c|c|c|c|c|c|c|}
\hline \multirow[t]{2}{*}{ Variables } & \multicolumn{3}{|c|}{ Cancer tissue } & \multicolumn{3}{|c|}{$\begin{array}{l}\text { Adjacent-to-carcinoma } \\
\text { tissue }\end{array}$} \\
\hline & $\overline{\mathrm{SSH} 1(+)}$ & $\mathrm{SSH} 1(-)$ & $P$-value & $\overline{\mathrm{SSH}} 1(+)$ & SSH1(-) & $P$-value \\
\hline Age (years) & & & 0.311 & & & 0.722 \\
\hline$\leq 55$ & 5 & 16 & & 15 & 2 & \\
\hline$>55$ & 29 & 50 & & 51 & 12 & \\
\hline Gender & & & 0.098 & & & 0.555 \\
\hline Male & 18 & 46 & & 40 & 7 & \\
\hline Female & 16 & 20 & & 26 & 7 & \\
\hline Location & & & 0.201 & & & 0.112 \\
\hline Sinuses & 16 & 41 & & 23 & 8 & \\
\hline Other & 18 & 25 & & 20 & 6 & \\
\hline $\begin{array}{l}\text { Lymph node } \\
\text { metastasis }\end{array}$ & & & $0.032^{*}$ & & & 0.461 \\
\hline Positive & 31 & 48 & & 53 & 10 & \\
\hline Negative & 3 & 18 & & 13 & 4 & \\
\hline $\begin{array}{l}\text { Positive lymph } \\
\text { node ratio }\end{array}$ & & & 0.201 & & & 0.112 \\
\hline$\geq 0.5$ & 18 & 25 & & 29 & 6 & \\
\hline$<0.5$ & 16 & 41 & & 37 & 8 & \\
\hline $\begin{array}{l}\text { Tumour } \\
\text { infiltration }\end{array}$ & & & 0.893 & & & 0.518 \\
\hline $\mathrm{T} 1$ & 2 & 6 & & 6 & 0 & \\
\hline $\mathrm{T} 2$ & 2 & 5 & & 2 & 3 & \\
\hline T3 & 23 & 41 & & 43 & 10 & \\
\hline $\mathrm{T} 4$ & 6 & 15 & & 14 & 2 & \\
\hline $\begin{array}{l}\text { Pathological } \\
\text { grading }\end{array}$ & & & 0.285 & & & 0.538 \\
\hline 2 & 4 & 11 & & 11 & 2 & \\
\hline 3 & 24 & 50 & & 48 & 9 & \\
\hline 4 & 6 & 5 & & 7 & 3 & \\
\hline Size & & & 0.181 & & & 0.137 \\
\hline$\geq 5 \mathrm{~cm}$ & 24 & 35 & & 40 & 5 & \\
\hline$<5 \mathrm{~cm}$ & 10 & 29 & & 26 & 9 & \\
\hline AJCC staging & & & 0.189 & & & 0.676 \\
\hline Stage I & 3 & 7 & & 5 & 2 & \\
\hline Stage II & 9 & 24 & & 25 & 4 & \\
\hline Stage III & 18 & 31 & & 30 & 6 & \\
\hline Stage IV & 4 & 3 & & 6 & 1 & \\
\hline
\end{tabular}

Additionally, there was no significant difference between SSH1 expression in adjacent-to-carcinoma tissues and clinico-pathological features of GC, including age, sex, tumour location, tumour size, pathological type, pathological grade, lymphatic metastasis and AJCC stage.

\section{Survival analysis}

Survival analysis showed that the median survival time of the 100 patients with GC was 39 months. KaplanMeier survival analysis demonstrated that the survival time in patients with SSH1-positive GC tissues was significantly correlated with prognosis $(P=0.016$; Fig. 2$)$. However, SSH1 expression in adjacent-to-carcinoma tissues was not significantly correlated with the prognosis of patients with GC in our study $(P=0.095$, Fig. 2$)$. Moreover, we found that some clinico-pathological parameters, including lymph node metastasis, positive lymph node ratio, pathological grading, tumour size, AJCC staging and tumour infiltration $(P=0.024, P<$ 0.001, $P=0.004, P=0.004, P<0.001, P=0.003$, respectively; Table 4 ), were significantly related to prognosis, while gender, age, tumour location and pathological type were not correlated with the prognosis of patients with GC (all $P>0.05$; data not shown).

The univariate analysis model showed that pathological grade, positive lymph node ratio, size, AJCC stage and SSH1 expression in cancer tissue were important factors for the predication of prognosis in patients with GC. All the variables that were statistically correlated with overall survival in the univariate analysis were included in the multivariate regression analysis. The results showed that positive lymph node ratio and SSH1 expression $(P=0.014$ and $P=0.030$, respectively) were prognostic factors influencing the survival of patients with GC (Table 4). These data clearly indicated that SSH1 expression is an exceptional predictor of poor prognosis for GC.

\section{Discussion}

Tumour development is a complex process, and multiple factors and steps participate in tumour malignant behaviour. The actin cytoskeleton plays important roles in cell migration, affecting such processes as cell-substrate adhesion, protrusion, phagocytosis and cytokinesis [12], and actin proteins form a large family and are central players in cell shape and movement [13]. SSH1mediated cofilin activation is an essential regulator of actin dynamics [14]; SSH1 and cofilin are highly expressed or highly activated in various malignant tumours, increasing the rate of invasion and metastasis of tumour cells through different mechanisms and pathways $[15,16]$. However, there is currently little evidence suggesting that SSH1 expression correlates with GC prognosis. The results of this study showed that SSH1 expression in GC tissues predicted GC lymph node metastasis based on TNM stage $(P=0.032$; Table 3$)$ and poor survival $(P=0.016$; Table $4 \&$ Fig. 2$)$. SSH1 expression is indeed a useful prognostic factor for GC and is significantly associated with a poor prognosis. 

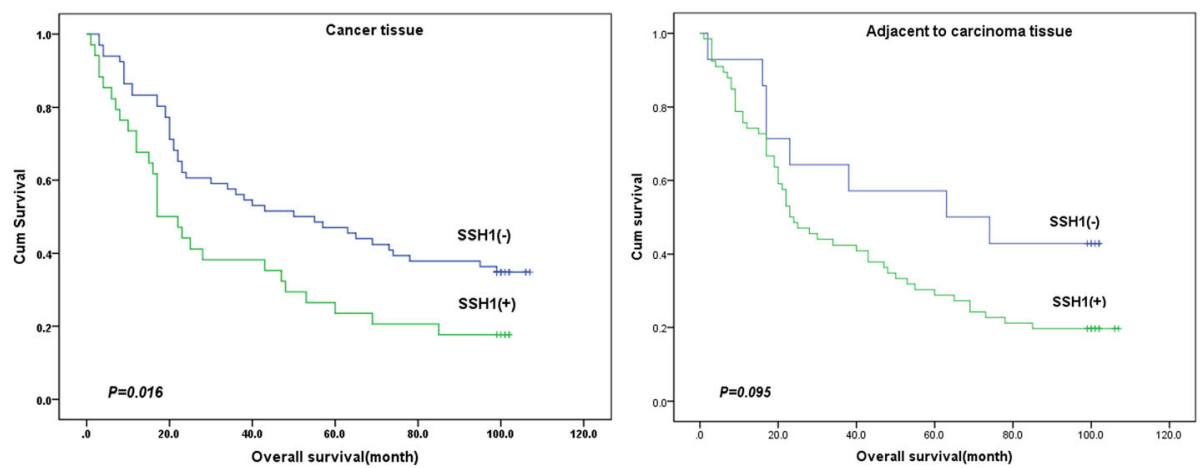

Fig. 2 Kaplan-Meier curves show that the survival time of patients with SSH1-positive GC and adjacent-to-carcinoma tissues correlated with prognosis

SSH1 dephosphorylates and activates cofilin, which is a member of the actin-depolymerizing factor/cofilin family of proteins $[12,17]$ that regulates formation of the actin cytoskeleton. Tumour invasion and metastasis, the main causes of cancer-related death, are directly associated with cofilin activity [18, 19]. Yusufu et al. [20] reported that the cofilin phosphorylation status plays a critical role in breast cancer, and dephosphorylated cofilin is related to lower overall survival. It has been reported that cofilin is related to GC progression [21]. Wang et al. [8] suggested that SSH1 may be a potential target to prevent or treat PC invasion and metastasis by regulating cofilin phosphorylation. Combined with our results, we speculated that SSH1 is a key protein that associates with F-actin, which plays a key role in the malignant biological behaviour of GC cells.

According to our IHC analysis and microarray data, SSH1 expression was not different between GC and adjacent-to-carcinoma tissues $(P=0.769$; Table 2$)$. However, in this study, SSH1 was expressed in GC tissue, and its expression level was positively correlated with tumour LNM and poor prognosis; multivariate regression analysis clearly indicated that SSH1 expression in GC tissues was an exceptional predictor of poor prognosis $(P=0.030$; Table 4). SSH1 affects tumour migration by altering cofilin activity, and researchers have proposed studying this interaction to gain better insight into the pathogenesis of digestive system tumours. Wang et al. [8] designed a study to explore the role of SSH1 in the development of PC; their results showed that SSH1 overexpression is related to cofilin activity and contributes to LNM and tumour cell migration, findings that were completely consistent with our results.

Not a single gene was activated within the migrating and invading tumour cells. Tumour invasion and metastasis, the main cause of cancer-related death, is directly associated with cofilin activity $[18,22]$. Tan [16] et al. reported that the poor prognosis of patients with colorectal cancer is related to regulation of $\mathrm{F}$-actin polymerization through the $\mathrm{SSH} /$ cofilin pathway. The

Table 4 Univariate and multivariate analyses of overall survival of GC patients

\begin{tabular}{|c|c|c|c|c|c|c|}
\hline \multirow[t]{2}{*}{ Variables } & \multicolumn{3}{|c|}{ Univariate analysis } & \multicolumn{3}{|c|}{ Multivariate analysis } \\
\hline & $\overline{H R}$ & $95 \% \mathrm{Cl}$ & $P$-value & $\overline{H R}$ & $95 \% \mathrm{Cl}$ & $P$-value \\
\hline Age (years) & 1.043 & $0.75-1.132$ & 0.288 & & & \\
\hline Gender & 1.142 & $0.952-1.378$ & 0.588 & & & \\
\hline Tumour Location & 1.024 & $0.944-1.389$ & 0.52 & & & \\
\hline Lymph node metastasis & 1.336 & $1.045-1.092$ & $0.024^{*}$ & 1.522 & $0.452-5.124$ & 0.472 \\
\hline Positive lymph node ratio & 2.584 & $1.267-7.321$ & $<0.001^{*}$ & 2.612 & $1.214-5.622$ & $0.014^{*}$ \\
\hline Pathological type & 1.018 & $0.694-1.258$ & 0.474 & & & \\
\hline Pathological grade & 2.094 & $1.316-4.167$ & $0.004^{*}$ & 0.474 & $0.153-1.464$ & 0.194 \\
\hline Tumour size & 1.921 & $1.156-3.126$ & $0.004^{*}$ & 0.367 & $0.049-2.752$ & 0.329 \\
\hline Tumour infiltration & 1.321 & $1.054-2.421$ & $0.003^{*}$ & 1.524 & $0.953-2.439$ & 0.079 \\
\hline AJCC staging & 2.126 & $1.335-6.075$ & $<0.001^{*}$ & 1.615 & $0.735-3.547$ & 0.233 \\
\hline Adjacent-to-GC tissue-SSH1 & 1.164 & $0.925-1.637$ & 0.095 & & & \\
\hline GC tissue-SSH1 & 1.564 & $1.109-2.352$ & $0.016^{*}$ & 1.787 & $1.056-3.021$ & $0.030^{*}$ \\
\hline
\end{tabular}


function of the SSH/cofilin signalling pathway in actin polymerization and dynamics was studied using small interfering RNA (siRNA) silencing of SSH1, down-regulation SSH1 led to decreases in vascular cell migration and metastatic progression $[9,23]$. Cell migration is associated with pathological remodelling at the site of vascular injury and with the invasive capacity of cancer cells. Our results showed that SSH1 expression level in $\mathrm{GC}$ was related to poor patient survival $(P=0.016)$. Therefore, SSH1 is the most important factor in cancer cell migration, and the abovementioned findings may indicate why the regulation of SSH1 activity is a promising therapeutic strategy to prolong the survival of cancer patients. As a regulator of cofilin, SSH1 may be an important regulatory factor in GC cell invasion and metastasis and may be closely related to the occurrence and development of GC. Therefore, the regulation of SSH1 activity is a promising therapeutic strategy for GC.

At present, there are three important limitations to our study. First, we used a retrospective design to analyse GC tissue samples from patients treated at a single centre and selection bias was unavoidable. Second, we analysed only the correlation with overall survival and could not access data regarding recurrence-free and disease-free survival. Third, our study did not include any molecular experiments.

\section{Conclusion}

In summary, this study clarified the clinical significance of SSH1 expression level in GC patients and showed that SSH1 activation plays an important role in GC progression and prognosis. Importantly, $\mathrm{SSH} 1$ dephosphorylates and activates cofilin, and SSH1 may play an important role in cell migration and cancer development through $\mathrm{SSH} /$ cofilin pathways. Our study indicated that SSH1 expression is a promising biomarker for the prevention and/or treatment of GC.

\section{Highlights}

1. Gastric cancer (GC) is a common type of malignancy and the second leading cause of cancer-related death in China [1]. Unfortunately, the prognosis for GC patients remains poor,therefore, it is important to identify biomarkers that are related to early diagnosis and prognosis.

2. Slingshot homolog-1 (SSH1) plays an important role in pathological processes, including in the occurrence and development of tumours [2-4] . However, there is currently no evidence suggesting that SSH1 expression correlates with GC prognosis. Thus, studies are needed to determine whether SSH1 is a prognostic biomarker for GC.
3. SSH1 is the most important factor in cancer cell migration, in our study clarified the clinical significance of SSH1 expression level in GC patients and showed that SSH1 activation plays an important role in $\mathrm{GC}$ progression and prognosis. As a regulator of cofilin, SSH1 may be an important regulatory factor in GC cell invasion and metastasis and may be closely related to the occurrence and development of GC.

Abbreviations

AJCC: American Joint Committee on Cancer; Cl: confidence interval; GC: Gastric cancer; HR: hazard ratio; IHC: immunohistochemistry;

PC: pancreatic cancer; SSH1: Slingshot homolog-1; TMA: tissue microarray

\section{Acknowledgements}

We would like to thank Zhenzhu Sun for help with evaluating the IHC staining. We would like to thank American Journal Experts (http:// www.aje.com) for language editing.

\section{Funding}

This work was supported by the National Natural Science Foundation of Xinjiang Uygur Autonomous Region (Grant No. 201554144).

Availability of data and materials

Data on this paper will be made available on reasonable request.

\section{Authors' contributions}

All authors contributed to the design of the study and writing of the manuscript. YM, MM, YL, SA, AAiniwaer, AAili, ZS and KA undertook the research and performed the analyses. All authors reviewed and approved the final version of the manuscript.

Ethics approval and consent to participate

This study was approved by the People's Hospital of Xinjiang Uygur Autonomous Region ethics committee(No.2006068), and written informed consent was received from the patients.

Consent for publication

Not applicable.

Competing interests

The authors declare that they have no competing interests.

\section{Publisher's Note}

Springer Nature remains neutral with regard to jurisdictional claims in published maps and institutional affiliations.

\section{Author details}

${ }^{1}$ Department of General Surgery (Research Institute of Minimally Invasive), People's Hospital of Xinjiang Uygur Autonomous Region, Urumai 830000, China. ${ }^{2}$ Department of Pathology, People's Hospital of Xinjiang Uygur Autonomous Region, Urumqi 830000, China.

Received: 23 December 2017 Accepted: 8 January 2018

Published online: 16 January 2018

References

1. Chen W, et al. Cancer statistics in China, 2015. CA Cancer J Clin. 2016;66: 115-32.

2. Fitzmaurice $C$, et al. Global, regional, and National Cancer Incidence, mortality, years of life lost, years lived with disability, and disability-adjusted life-years for 32 cancer groups, 1990 to 2015: a systematic analysis for the global burden of disease study. JAMA Oncol. 2017;3:524-48.

3. Cidon EU, Ellis SG, Inam Y, et al. Molecular targeted agents for gastric cancer: a step forward towards personalized therapy[J]. Cancers. 2013; 5:64-91. 
4. Steeg PS. Metastasis suppressors alter the signal transduction of cancer cells. Nat Rev Cancer. 2003;3:55-63.

5. Sarmiere PD, Bamburg JR. Regulation of the neuronal actin cytoskeleton by ADF/cofilin. J Neurobiol. 2004:58:103-17.

6. Niwa R, et al. Control of actin reorganization by slingshot, a family of phosphatases that dephosphorylate ADF/cofilin. Cell. 2002;108:233-46.

7. Nishita M, et al. Phosphoinositide 3-kinase-mediated activation of cofilin phosphatase slingshot and its role for insulin-induced membrane protrusion. J Biol Chem. 2004;279:7193-8.

8. Wang $Y$, et al. Cofilin-phosphatase slingshot-1L (SSH1L) is over-expressed in pancreatic cancer (PC) and contributes to tumor cell migration. Cancer Lett. 2015;360:171-6.

9. Torres RA, et al. Slingshot isoform-specific regulation of cofilin-mediated vascular smooth muscle cell migration and neointima formation. Arterioscler Thromb Vasc Biol. 2011:31:2424-31.

10. Michiru Nishita CT, Yamamoto M, Horita Y, Ohashi K, Mizuno K. Spatial and temporal regulation of cofilin activity by LIM kinase and slingshot is critical for directional cell migration. J Cell Biol. 2005;171:349-59.

11. Yang J, Long $\mathrm{Q}, \mathrm{Li} \mathrm{H}$, et al. The value of positive lymph nodes ratio combined with negative lymph node count in prediction of breast cancer survival[]]. J Thorac Dis. 2017;9:1531-7.

12. Bamburg JR. Proteins of the ADF/cofilin family: essential regulators of actin dynamics. Annu Rev Cell Dev Biol. 1999;15:185-230.

13. Pollard TD, Cooper JA. Actin, a central player in cell shape and movement. Science. 2011;326:1208-12.

14. Lappalainen P, Drubin DG. Cofilin promotes rapid actin filament turnover in vivo. Nature. 1997;388:78-82

15. Zhang YT, et al. Formation of cofilin-actin rods following cucurbitacin-Binduced actin aggregation depends on slingshot homolog 1-mediated cofilin hyperactivation. J Cell Biochem. 2013;114:2415-29.

16. Tan F, et al. Neuron navigator 2 overexpression indicates poor prognosis of colorectal cancer and promotes invasion through the SSH1L/cofilin-1 pathway. J Exp Clin Cancer Res. 2015;34:117.

17. Takahashi K, Okabe H, Kanno Sl, et al. A pleckstrin homology-like domain is critical for F-actin binding and cofilin-phosphatase activity of Slingshot-1.[J]. Biochem Biophys Res Commun. 2017;482:686-92.

18. Sidani $\mathrm{M}$, et al. Cofilin determines the migration behavior and turning frequency of metastatic cancer cells. J Cell Biol. 2007;179:777-91.

19. Ghosh M, et al. Cofilin promotes Actin polymerization and defines the direction of cell motility. Science. 2004;304:743-6.

20. Maimaiti Y, et al. Dephosphorylated cofilin expression is associated with poor prognosis in cases of human breast cancer: a tissue microarray analysis. Oncotargets Ther. 2016;9:6461-6.

21. Li D, et al. Activated Pak4 expression correlates with poor prognosis in human gastric cancer patients. Tumor Biol. 2015;36:9431-6.

22. Wang $W$, Mouneimne $G$, Sidani $M$, et al. The activity status of cofilin is directly related to invasion, intravasation, and metastasis of mammary tumors [J]. J Cell Biol. 2006:173(3):395-404.

23. Horita $Y$, et al. Suppression of the invasive capacity of rat ascites hepatoma cells by knockdown of slingshot or LIM kinase. J Biol Chem. 2008;283:6013-21.

\section{Submit your next manuscript to BioMed Central and we will help you at every step:}

- We accept pre-submission inquiries

- Our selector tool helps you to find the most relevant journal

- We provide round the clock customer support

- Convenient online submission

- Thorough peer review

- Inclusion in PubMed and all major indexing services

- Maximum visibility for your research

Submit your manuscript at www.biomedcentral.com/submit

) Biomed Central 